\title{
Assessment of the effect of a Salmonella enterica ser. Typhimurium culture supernatant on the single-cell lag time of foodborne pathogens
}

\author{
Vasiliki A. Blana ${ }^{1}$, Alexandra Lianou ${ }^{*, 1}$, George-John E. Nychas

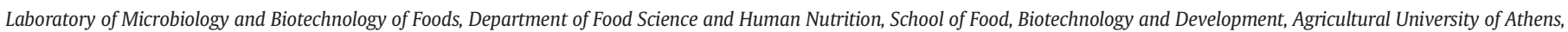
Athens 11855, Greece

\section{A R T I C L E I N F O}

\section{Article history:}

Received 7 May 2015

Received in revised form 5 September 2015

Accepted 11 September 2015

Available online 13 September 2015

\section{Keywords:}

Autoinducer-2

Lag time

Quorum sensing

Salmonella enterica

Single-cell kinetics

Staphylococcus aureus

\begin{abstract}
A B S T R A C T
The objective of this study was the in vitro evaluation of the effect of a cell-free microbial supernatant, produced by a luxS-positive Salmonella enterica ser. Typhimurium strain, on the single-cell growth kinetic behavior of two strains of S. enterica (serotypes Enteritidis and Typhimurium) and a methicillin-resistant Staphylococcus aureus strain. The single-cell lag time $(\lambda)$ of the pathogens was estimated in the absence and presence $(20 \% v / v)$ of microbial supernatant based on optical density measurements. As demonstrated by the obtained results, the tested microbial supernatant had a strain-specific effect on the single-cell $\lambda$ and its variability. Although the mean $\lambda$ values were similar in the absence and presence of microbial supernatant in the case of Salmonella Enteritidis, a significant $(P \leq 0.05)$ reduction and increase in the mean value of this parameter in the presence of microbial supernatant were observed for Salmonella Typhimurium and St. aureus, respectively. With regard to the effect of the tested microbial supernatant on the single-cell variability of $\lambda$, similar $\lambda$ distributions were obtained in its absence and presence for $S$. Enteritidis, while considerable differences were noted for the other two tested organisms; the coefficient of variation of $\lambda$ in the absence and presence of microbial supernatant was 41.6 and $69.8 \%$ for $S$. Typhimurium, respectively, with the corresponding values for St. aureus being 74.0 and $56.9 \%$. As demonstrated by the results of bioassays, the tested microbial supernatant exhibited autoinducer-2 activity, indicating a potential association of such quorum sensing compounds with the observed effects. Although preliminary in nature, the collected data provide a good basis for future research on the role of quorum sensing in the single-cell growth behavior of foodborne pathogens.
\end{abstract}

(c) 2015 Elsevier B.V. All rights reserved.

\section{Introduction}

In nature, the presence, survival and proliferation of microorganisms in multicellular communities are, to a great extent, delineated by microbial interactions taking place at both intra- and inter-species levels (Duan et al., 2009). Various chemical compounds, produced as part of bacterial metabolism and diffused and accumulated in the surrounding growth environment, have been shown to be involved in such interactions and determine their character (i.e. neutral, cooperative or competitive microbial relationships) (Duan et al., 2009). In this context, spent culture supernatants (or conditioned media) have been frequently utilized for the purpose of evaluating the effect of microbial products on bacterial behavior, with the ultimate goal of elucidating microbial interactions and/or identifying natural antimicrobial compounds

\footnotetext{
* Corresponding author at: Department of Food Science \& Human Nutrition, Laboratory of Microbiology and Biotechnology of Foods, Agricultural University of Athens, Iera Odos 75, Athens 11855, Greece.

E-mail addresses: alianou@aua.gr (A. Lianou), gjn@aua.gr (G.-J.E. Nychas).

1 These authors contributed equally to this study.
}

(Chorianopoulos et al., 2010; Dourou et al., 2011; Lopes et al., 2011; Qin et al., 2009).

Cell-to-cell communication has been widely identified as a process of strong implication in bacterial gene regulation and of major significance for microbial interactions (Waters and Bassler, 2005). The system underlying cell-to-cell communication is known as quorum sensing (QS) and involves the production, release and subsequent detection of and response to chemical signal molecules referred to as autoinducers (Fuqua et al., 1994). Among the four so far identified groups of microbial QS compounds, the furanosyl borate diesters, encoded by the luxS gene and referred to as autoinducer-2 (AI-2) molecules, stand out due to their ability to serve as universal chemical signals, being involved in both intra- and inter-species communication (Federle and Bassler, 2003). In the context of QS, various cellular functions and bacterial traits are modulated in response to threshold autoinducer concentrations including bioluminescence, transfer of conjugative plasmids, virulence, symbiosis, competence, secretion of nutrient-sequestering compounds, synthesis of antimicrobial compounds and biosurfactants, motility, sporulation and biofilm formation (Jesudhasan et al., 2010; Nadell et al., 2008). With particular reference to food microbiology, numerous studies have investigated the role of QS in the virulence potential of 
foodborne pathogens, as well as in the biofilm-forming ability of both food spoilage and pathogenic bacteria (Painter et al., 2014; Skandamis and Nychas, 2012). Nevertheless, limited research studies have assessed the involvement of QS in the growth behavior of microorganisms in vitro (Dourou et al., 2011; Dunstall et al., 2005; Nychas et al., 2009) or in situ (Rasch et al., 2005; Wevers et al., 2009). Furthermore, the available research data refer to microorganisms being studied exclusively at a population level, with the potential variability in the behavior of individual bacterial cells not being taken into account.

Given the above, the objective of the present study was the evaluation of the effect of a microbial supernatant, produced by a luxS-positive Salmonella enterica ser. Typhimurium strain, on the single-cell lag time of two important foodborne pathogens, namely S. enterica and Staphylococcus aureus.

\section{Materials and methods}

\subsection{Bacterial strains and culture conditions}

The bacterial strains used in the present study, along with all pertinent information (designation, origin, source and exact use in the conducted experiments), are presented in Table 1. Stock cultures of the strains were stored frozen $\left(-80^{\circ} \mathrm{C}\right)$ onto treated beads in a cryoprotective fluid (Protect Microorganism Preservation System, Lab M Limited, Lancashire, UK).

With regard to the $S$. enterica and St. aureus strains, frozen stock cultures were revived by transferring one bead in $10 \mathrm{ml}$ of tryptone soy broth (TSB; Lab M Limited) and incubating at $37^{\circ} \mathrm{C}$ for $24 \mathrm{~h}$, and working cultures were stored refrigerated $\left(4^{\circ} \mathrm{C}\right)$ on tryptone soy agar (TSA; Lab M Limited) and were renewed biweekly. For the purpose of inoculum preparation, these strains were activated by transferring a single colony from TSA into $10 \mathrm{ml}$ of Luria-Bertani broth (Bertani, $1951)$ supplemented with $0.5 \%(w / v)$ glucose (Merck, Darmstadt, Germany) (LBG), and incubating with agitation (160 rpm) at $37^{\circ} \mathrm{C}$ for $24 \mathrm{~h}$. The pH of LBG broth used for strain activation was adjusted to 6.5 with $10 \mathrm{~N} \mathrm{HCl}$ (Merck) using a digital pH meter (RL150, Russell $\mathrm{pH}$, Cork, Ireland) with a glass electrode (Metrohm AG, Herisau, Switzerland). Portions (10- $\mu \mathrm{l}$ ) of the activated cultures were transferred into $10 \mathrm{ml}$ of fresh LBG broth of the above properties and incubated with agitation (160 rpm) at $37{ }^{\circ} \mathrm{C}$ for $8 \mathrm{~h}$ (S. Typhimurium CDC 6516-60) or $18 \mathrm{~h}$ (S. Enteritidis, $S$. Typhimurium 4/74 and St. aureus).

With reference to the Vibrio harveyi strains, frozen stock cultures were revived by transferring one bead in $10 \mathrm{ml}$ of autoinducer bioassay $(A B)$ broth (Lu et al., 2004) and incubating with agitation (160 rpm) at $30{ }^{\circ} \mathrm{C}$ for $24 \mathrm{~h}$, while working cultures were stored refrigerated $\left(4^{\circ} \mathrm{C}\right)$ onto $\mathrm{AB}$ agar and renewed biweekly. The cultures used in the $\mathrm{AI}-2$ activity bioassay (described in Section 2.3) were prepared by transferring a single colony from $A B$ agar in $10 \mathrm{ml}$ of $A B$ broth, and incubating with agitation $(160 \mathrm{rpm})$ at $30^{\circ} \mathrm{C}$ for $16 \mathrm{~h}$.

\subsection{Preparation of microbial supernatants}

The microbial supernatant evaluated in this study was produced from late-exponential-phase cultures (i.e. 8-h cultures in LBG of $\mathrm{pH} 6.5$ as described above) of the luxS-positive S. Typhimurium strain CDC 6516-60 (Table 1). More specifically, bacterial cells were removed from the growth medium via centrifugation (Heraus Multifuge 1S-R, Thermo Electron Corporation, Langenselbold, Germany) at $5000 \times \mathrm{g}$ for $15 \mathrm{~min}$ at $4{ }^{\circ} \mathrm{C}$, followed by filtration through $0.2 \mu \mathrm{m}$-pore-size filters (Whatman, Inc., Clifton, NJ, USA). Part of the produced microbial supernatant was heat treated $\left(121^{\circ} \mathrm{C}\right.$ for $\left.15 \mathrm{~min}\right)$, and both types of cell-free culture supernatants (i.e. heat treated and untreated) were maintained at $4{ }^{\circ} \mathrm{C}$ until use (maximum storage period of 4 days).

\subsection{AI-2 activity bioassays}

The AI-2 activity of the tested microbial supernatants, as well as of cell-free culture supernatants of the tested (for their growth behavior) S. enterica and St. aureus strains, was evaluated using the biosensor $V$. harveyi BAA-1117 strain (Table 1) and the procedure described by Surette and Bassler (1998). Sterile growth medium (LBG of pH 6.5) and microbial supernatant obtained from the AI-2-producing $V$. harveyi BAA-1119 (Table 1) strain were used as negative and positive control, respectively. Luminescence measurements were taken using a Synergy HT multi-mode microplate reader (Biotek ${ }$, Winooski, VT, USA), and AI-2 activity was expressed as "relative AI-2 activity" (i.e. ratio of the luminescence of the sample containing the microbial supernatant under evaluation to that of the negative control).

\subsection{Growth experiments}

The growth behavior of the tested $S$. Enteritidis, S. Typhimurium 4/74 and St. aureus strains was evaluated in LBG broth ( $\mathrm{pH}$ 6.5) containing: (i) no microbial supernatant (Control); (ii) 20\% ( $v / v)$ microbial supernatant (MS); and (iii) 20\% ( $v / v)$ microbial supernatant that was subjected to heat treatment (MS-HT), as described in Section 2.2. The 18-h culture of each one of the strains was centrifuged (Heraeus Multifuge $1 \mathrm{~S}-\mathrm{R}$, Thermo Electron Corporation) at $5000 \times \mathrm{g}$ for $10 \mathrm{~min}$ at $4{ }^{\circ} \mathrm{C}$. The harvested cells were washed with quarter strength Ringer's solution (Lab M Limited) and centrifuged under the same conditions. The harvested cells of the washed cultures were finally resuspended in $10 \mathrm{ml}$ of LBG of the above characteristics (Control, MS or MS-HT), and 300- $\mu \mathrm{l}$ aliquots of appropriate dilution(s) (conducted in the same medium) were transferred in 96-well polystyrene microplates (Nuova Aptaca S.r.l., Canelli, Italy). The growth kinetic parameters of the tested strains were estimated from optical density (OD) measurements, with the latter being taken at $600 \mathrm{~nm}$ using the Synergy HT automated spectrophotometer. The microplates were placed in the spectrophotometer at an incubation temperature of $25^{\circ} \mathrm{C}$, and OD measurements were

Table 1

Bacterial strains used in this study and their characteristics.

\begin{tabular}{|c|c|c|c|c|}
\hline Bacterial strain & Designation & Description & Origin & Reference or source \\
\hline Salmonella enterica ser. Enteritidis PT4 & P167807 & Tested strain ${ }^{1}$ & Food isolate (eggs) & $\begin{array}{l}\text { Division of Enteric Pathogens, Central } \\
\text { Public Health Laboratory, London, UK }\end{array}$ \\
\hline S. enterica ser. Typhimurium & $4 / 74$ & Tested strain ${ }^{1}$ & Calf isolate (bowel) & Hoiseth and Stocker, 1981 \\
\hline S. enterica ser. Typhimurium & $\begin{array}{l}\text { CDC 6516-60 } \\
(\text { ATCC 14028) }\end{array}$ & $\begin{array}{l}\text { Strain used in microbial supernatant } \\
\text { production; luxS-positive }\end{array}$ & Chicken isolate (tissue) & Tindall et al., 2005 \\
\hline Staphylococcus aureus (methicillin-resistant) & $\mathrm{COL}$ & Tested strain ${ }^{1}$ & Clinical isolate & Gill et al., 2005 \\
\hline Vibrio harveyi & $\begin{array}{l}\text { BAA-1117 } \\
(\text { ATCC BB-170) }\end{array}$ & Biosensor strain $^{2}$ & - & Bassler et al., 1997 \\
\hline Vibrio harveyi & $\begin{array}{l}\text { BAA-1119 } \\
(\text { ATCC BB152) }\end{array}$ & AI-2 producer (bioassay positive control) & - & Bassler et al., 1997 \\
\hline
\end{tabular}

\footnotetext{
1 Bacterial strains evaluated for their growth behavior.

2 The strain contains luxN:Tn5, bioluminescent reporter, and cognate signal: borated AI-2.
} 
taken at 15-min intervals after agitation of the microplates for $10 \mathrm{~s}$ at medium amplitude.

\subsection{Maximum specific growth rate}

The maximum specific growth rate $\left(\mu_{\max }\right)$ of each strain in each one of the tested treatments was estimated using the decimal dilution approach, as previously described (Aguirre et al., 2011; Lianou and Koutsoumanis, 2011). In the context of this approach, $\mu_{\max }$ is estimated from absorbance detection times ( $\left.T_{\mathrm{det}}\right)$ of serially decimally diluted cultures, with $T_{\text {det }}$ being defined as the time required for a certain OD measurement (or increase) to be observed. In this study, six serial decimal dilutions of each culture were made in LBG of the same characteristics with the corresponding treatment, and then, 300- $\mu$ l aliquots of each dilution were dispensed in the microplates (sixteen replicate wells for each dilution), resulting in initial bacterial concentrations ranging approximately from $10^{6}$ to $10 \mathrm{CFU} /$ well. The $T_{\mathrm{det}}$ was defined as the time required for an OD measurement of 0.2 to be obtained (corresponding to a bacterial concentration of $\mathrm{ca}$. $10^{7} \mathrm{CFU} /$ well), while a mean value (of the sixteen replicate samples) was estimated for each dilution. Furthermore, dilutions of each culture were surface plated on TSA and colonies were counted after incubation of the plates at $37^{\circ} \mathrm{C}$ for $24 \mathrm{~h}$. The $T_{\mathrm{det}}$ values of the serial decimal dilutions were plotted against the natural logarithm of the corresponding bacterial concentration for each tested strain (ln CFU/well), and $\mu_{\max }$ values were determined by linear regression, using Microsoft ${ }^{\circledR}$ Excel, according to the following equation:

$\ln (N)=k-\mu_{\max } \cdot T_{\text {det }}$

Three independent experiments were conducted for each one of the tested bacterial strains and treatments.

\subsection{Lag time}

The single-cell lag time $(\lambda)$ values of the tested organisms were estimated based on turbidity growth curves, according to a widely used methodology (Aguirre et al., 2011; Baranyi and Pin, 1999; Baranyi et al., 2009).

The culture of each one of the tested strains was decimally diluted in the treatment medium (Control, MS or MS-HT) to a concentration of ca. $10^{\circ} \mathrm{CFU} / \mathrm{ml}$, and $300-\mu \mathrm{l}$ aliquots of this dilution were dispensed in the 96 wells of a microplate. The average number of cells per microplate well was estimated based on the assumption that the probability of having one cell per well is described by the Poisson distribution (Baranyi et al., 2009). More specifically, the equation connecting the average number of cells per well $(m)$ with the probability of no growth occurring in a well $\left(P_{0}\right)$ is the following:

$m=-\operatorname{Ln} P_{0}$

with $m$ being obtained, based on the calculated $P_{0}$ values, from Poisson distribution tables. Given that $P_{0}$ values less than 0.14 or higher than 0.85 are expected to result in inaccurate or unreliable single-cell $\lambda$ estimates, respectively (Aguirre et al., 2011), only data corresponding to wells of $m$ lower than 2 and higher than 0.36 were taken into account.

In this context, single-cell $\lambda$ values were estimated using the following equation (Baranyi and Pin, 1999):

$\lambda=T_{\mathrm{det}}-\frac{\operatorname{Ln}\left(N_{\mathrm{det}}\right)-\operatorname{Ln}\left(N_{0}\right)}{\mu_{\max }}$

where $N_{\text {det }}$ is the bacterial concentration at $T_{\text {det }}, N_{0}$ is the number of cells initiating growth in each microplate well, and $\mu_{\max }$ is the maximum specific growth rate of each bacterial strain estimated as described in Section 2.5
The experiments were replicated as many times needed for at least 150 values of $\lambda$ to be obtained for each one of the tested bacterial strains and growth media.

\subsection{Statistical analysis}

The estimated $\mu_{\max }$ and $\lambda$ values were evaluated by analysis of variance using the general linear model procedure of the IBM SPSS Statistics for Windows software, Version 22.0 (IBM Corp., Armonk, NY, USA). Homogeneity of variance was assessed using the Levene's test, and means were separated using the Tukey HSD or the Games-Howell test in the case of homogeneous or significantly different variances, respectively, at a significance level of $\alpha=0.05$.

\section{Results and discussion}

Based on the bioassays' results, both the non-diluted (100\% $v / v)$ and diluted $(20 \% \mathrm{v} / \mathrm{v})$ microbial supernatants, produced by $S$. Typhimurium CDC 6516-60, expressed substantial AI-2 activity, which was 79.2 and 37.3-fold higher than that of the negative control (i.e. sterile LBG broth), respectively (Fig. 1). Such finding was anticipated, given that the strain used for the supernatant production is luxS-positive (Jesudhasan et al., 2010), and the luxS/AI-2 system is one of the two QS systems used by S. enterica for the purpose of intercellular signaling (Surette and Bassler, 1998; Taga et al., 2003). On the other hand, the corresponding relative AI-2 activity of MS-HT was similar to that of the negative control, indicating that the intended inactivation of AI-2 signal molecules (Surette and Bassler, 1998) was, most likely, provided by the applied heat treatment. The indigenous ability of the three tested (for their growth behavior) bacterial strains to express AI-2 activity also was evaluated and, as illustrated in Fig. 2, both of the tested S. enterica strains exhibited significant such activity. On the contrary, the relative AI-2 activity of the St. aureus supernatant was not considerably different from that of the negative control under the conditions of this study (Fig. 2).

As demonstrated by the estimated $\mu_{\max }$ values (Table 2 ), no significant effect of the microbial supernatant on this growth kinetic parameter could be observed, with the parameter's values being similar among the three tested treatments for both S. enterica and St. aureus strains. On the other hand, the effect of microbial supernatant on the single-cell $\lambda$ appeared to depend on the tested microbial species and strain (Table 3). With regard to $S$. enterica, a different behavior between the two tested strains was observed in response to the presence of microbial supernatant in the growth medium. Although the mean single-cell $\lambda$ values were similar for the Control and MS treatments in the case of $S$. Enteritidis, a considerable reduction $(P \leq 0.05)$ in the mean value of this parameter in MS $\left(1.81 \mathrm{~h}^{-1}\right)$ compared to the Control

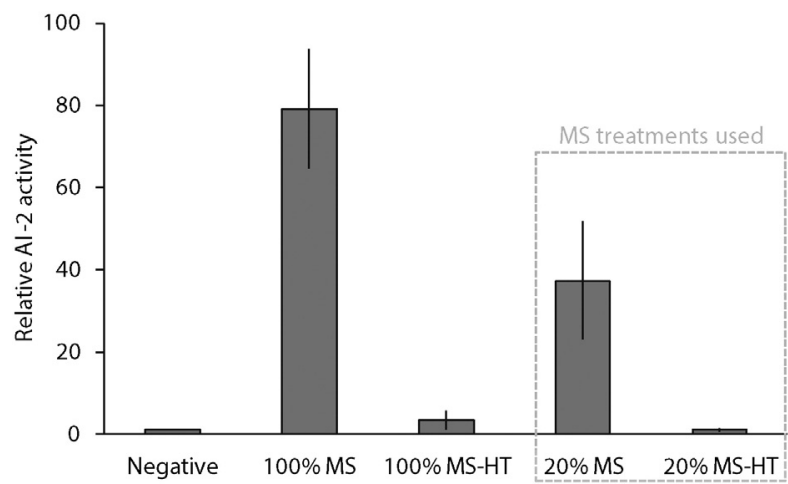

Fig. 1. Mean ( \pm standard deviation) relative AI-2 activity of 20 and $100 \%(v / v)$ microbial supernatant (MS) or microbial supernatant subjected to heat treatment (MS-HT), obtained from 8-h culture (in Luria-Bertani broth supplemented with $0.5 \%(w / v)$ glucose, LBG; $37^{\circ} \mathrm{C}$ ) of Salmonella enterica ser. Typhimurium (CDC 6516-60). Sterile LBG broth was used as negative control. 


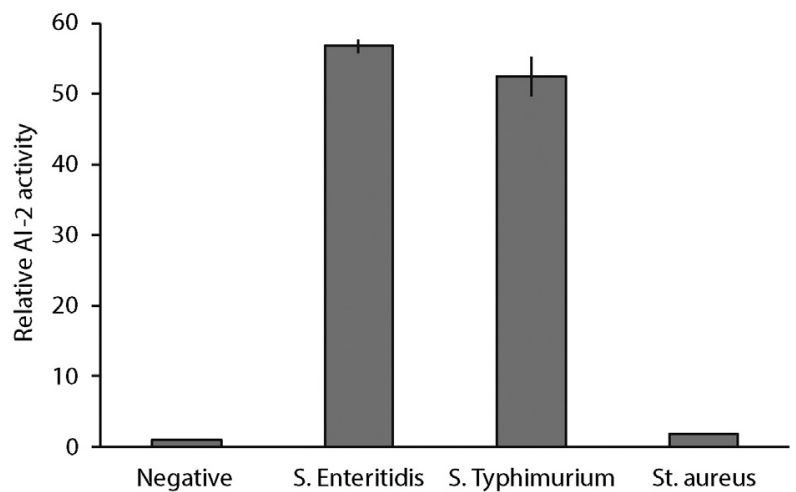

Fig. 2. Mean ( \pm standard deviation) relative $\mathrm{AI}-2$ activity of microbial supernatants obtained from 8-h cultures (in Luria-Bertani broth supplemented with $0.5 \%(w / v)$ glucose, LBG; $37^{\circ} \mathrm{C}$ ) of Salmonella enterica ser. Enteritidis, S. enterica ser. Typhimurium (4/74) and Staphylococcus aureus. Sterile LBG broth was used as negative control.

$\left(2.26 \mathrm{~h}^{-1}\right)$ was observed for $S$. Typhimurium (Table 3$)$. Such observation may, to some extent, be associated with the fact that the latter tested strain is of the same serotype (i.e. Typhimurium) as the strain used for the production of the applied microbial supernatant. In contrast to the observations made for S. Typhimurium, the single-cell $\lambda$ of St. aureus was significantly $(P \leq 0.05)$ longer in MS compared to the Control, with the mean parameter's values being 9.48 and $5.92 \mathrm{~h}$, respectively (Table 3).

Despite the fact that the presence of QS signal molecules in food systems has been demonstrated (Blana and Nychas, 2014; Blana et al., 2011; Nychas et al., 2009), their exact role has not been completely exemplified. The majority of studies on cell-to-cell communication have focused on assessing the molecular aspects and the genetic processes underlying this phenomenon (Surette and Bassler, 1998; Taga et al., 2003), with relatively limited attention being paid to how both intraand inter-species communication modulate bacterial growth responses. Indeed, the findings of relatively few studies have suggested a considerable effect of QS compounds on the growth behavior of food spoilage or pathogenic bacteria, either in vitro (Dourou et al., 2011; Dunstall et al., 2005; Kolling and Matthews, 2007; Nychas et al., 2009; Whan et al., 2000) or in situ (Rasch et al., 2005; Wevers et al., 2009), with their results being often contradictory and inconclusive.

Similar with the observations made for $S$. Typhimurium in the present study were the findings of Kolling and Matthews (2007), who reported that AI-2 signaling compounds present in the cell-free culture supernatant of Escherichia coli 0157:H7 significantly enhanced the growth of stressed cells of the pathogen. Likewise, according to the findings of Nychas et al. (2009), the growth rate of Pseudomonas fluorescens, estimated through monitoring of conductance changes in the growth medium, was enhanced in the presence of cell-free meat extract derived

\section{Table 2}

Maximum specific growth rate $\left(\mu_{\max }\right)$ values of Salmonella enterica and Staphylococcus aureus strains in Luria-Bertani broth supplemented with $0.5 \%(\mathrm{w} / \mathrm{v})$ glucose at $25^{\circ} \mathrm{C}$, in the absence (Control) and presence of microbial supernatant (MS) containing autoinducer-2 signal molecules.

\begin{tabular}{lll}
\hline Bacterium & Treatment & $\mu_{\max }\left(\mathrm{h}^{-1}\right)^{2}$ \\
\hline \multirow{3}{*}{ Salmonella Enteritidis } & Control & $0.94 \pm 0.08$ \\
& MS & $0.97 \pm 0.09$ \\
Salmonella Typhimurium & MS-HT & $0.91 \pm 0.10$ \\
& Control & $0.98 \pm 0.09$ \\
Staphylococcus aureus & MS & $0.93 \pm 0.06$ \\
& MS-HT & $1.04 \pm 0.13$ \\
& Control & $0.41 \pm 0.07$ \\
& MS & $0.43 \pm 0.04$ \\
\hline
\end{tabular}

\footnotetext{
1 Microbial supernatant subjected to heat treatment $\left(121^{\circ} \mathrm{C}, 15 \mathrm{~min}\right)$.

2 Values are means \pm standard deviations $(n=3)$.
}

\section{Table 3}

Lag time of single bacterial cells in Luria-Bertani broth supplemented with $0.5 \%(w / v)$ glucose at $25{ }^{\circ} \mathrm{C}$, in the absence (Control) and presence of microbial supernatant (MS) containing autoinducer-2 signal molecules.

\begin{tabular}{clllll}
\hline Bacterium & Treatment & $\begin{array}{l}\text { No. } \\
\text { samples }^{2}\end{array}$ & $\begin{array}{l}\text { No. } \\
\text { cells/well } \\
(\text { min-max })^{3}\end{array}$ & Lag time $(\mathrm{h})^{4}$ & $\begin{array}{l}\text { Coefficient } \\
\text { of variation } \\
(\%)\end{array}$ \\
\hline Salmonella & Control & 193 & $0.37-0.65$ & $3.68 \pm 0.89 \mathrm{~A}$ & 24.3 \\
Enteritidis & MS & 210 & $0.37-0.94$ & $3.49 \pm 0.99 \mathrm{~A}$ & 28.2 \\
& MS-HT & 170 & $0.43-0.78$ & $3.00 \pm 0.91 \mathrm{~B}$ & 30.2 \\
Salmonella & Control & 159 & $0.71-0.89$ & $2.26 \pm 0.94 \mathrm{~B}$ & 41.6 \\
Typhimurium & MS & 202 & $0.67-0.99$ & $1.81 \pm 1.26 \mathrm{C}$ & 69.8 \\
Staphylococcus & MS-HT & 221 & $0.78-0.92$ & $3.95 \pm 1.15 \mathrm{~A}$ & 29.1 \\
aureus & Control & 180 & $0.42-1.47$ & $5.92 \pm 4.38 \mathrm{~B}$ & 74.0 \\
& MS & 195 & $0.42-1.43$ & $9.27 \pm 5.27 \mathrm{~A}$ & 56.9 \\
& MS-HT & 177 & $0.82-1.17$ & $9.48 \pm 5.38 \mathrm{~A}$ & 56.8 \\
\hline
\end{tabular}

1 Microbial supernatant subjected to heat treatment $\left(121^{\circ} \mathrm{C}, 15 \mathrm{~min}\right)$.

2 Total number of microplate wells showing growth (all replicates included).

3 Average number of cells per microplate well, as obtained from Poisson distribution tables (range of values calculated for the different replicates).

${ }^{4}$ Values are means \pm standard deviations. Within a column and for each bacterium, means lacking a common letter are significantly different $(P<0.05)$.

from spoiled meat and containing QS compounds. Monitoring of the effect of QS signal molecules, present in the cell-free culture supernatants of various microorganisms, on the growth and metabolic activity of $S$. Enteritidis and $S$. Typhimurium indicated that such effect depended on the bacterial strain producing the signal molecules (Dourou et al., 2011). As demonstrated by the results of the present study, where a single strain was used for the production of the tested microbial supernatant, the observed effect of this supernatant on the growth kinetic behavior of $S$. enterica (in terms of single-cell $\lambda$ ) also depended on the tested pathogen's strain. Since a significant effect was only observed for the strain belonging to serotype Typhimurium, and not for the strain of serotype Enteritidis (Table 3), an association of the pathogen's serotype with the observations made may exist. However, given that only two $S$. enterica strains were evaluated in this study, and that the majority of the available research data, pertinent to the QS systems of the pathogen, refer to serotype Typhimurium (Jesudhasan et al., 2010; Surette and Bassler, 1998; Taga et al., 2003), there is not enough information to firmly support a serotype-specific behavior.

Although the main well-characterized QS system in staphylococci is the "accessory gene regulator" (agr), which has been shown to play a central role in staphylococcal pathogenesis (Atkinson and Williams, 2009), a second QS system, dependent on the AI-2 synthase gene luxS, has also been described (Federle and Bassler, 2003). As mentioned above, the microbial supernatant produced by the tested St. aureus strain did not exhibit AI-2 activity (Fig. 2). Nonetheless, given that AI-2 signal molecules are considered to be universal, allowing bacteria to respond not only to endogenously produced AI-2 signals but also to those produced by other bacterial species in their vicinity (Federle and Bassler, 2003), it is interesting to assess the responses of this pathogen, regardless of its ability to produce such molecules on its own. Based on the results of the present study, the single-cell $\lambda$ of the tested St. aureus strain was significantly increased in the presence of microbial supernatant expressing AI-2 activity (Table 3 ). In the absence of any data specifically related to growth, the above observation can only be discussed in conjunction with similar findings relevant to biofilm formation and virulence. Unlike many QS systems described in Gramnegative bacteria, both agr and luxS of staphylococci have been shown to reduce rather than induce biofilm formation and virulence (Kong et al., 2006; Yu et al., 2012). For instance, luxS has been shown to reduce cell-to-cell adhesion of St. aureus by down-regulating the expression of biofilm exopolysaccharides (Kong et al., 2006). In any case, the expression, under the conditions of this study, of luxS in the tested St. aureus strain needs to be determined if solid conclusions regarding its potential for AI-2 activity and the involvement of the latter in its growth behavior are to be drawn. 
Besides the overall effect of the tested microbial supernatant on the pathogens' growth, as this can be evaluated based on the mean values of the single-cell $\lambda$, it is also important to look into the corresponding effect on the single-cell variability of this growth parameter. As demonstrated by the values of the percent coefficient of variation (CV $=100 \times$ standard deviation / mean), the effect of the applied microbial supernatant on the variability of $\lambda$ was different for the three strains studied (Table 3). Although no considerable differences in the CV values were observed for $S$. Enteritidis, the single-cell variability of $\lambda$ was substantially increased in MS compared to the Control in the case of $S$. Typhimurium, with the estimated values of CV being 69.8 and $41.6 \%$, respectively. On the other hand, the variability of $\lambda$ of St. aureus decreased in the presence of microbial supernatant, with the $\mathrm{CV}$ being reduced from $74.0 \%$ in the Control to $56.9 \%$ in the MS treatment (Table 3). A similar conclusion can be drawn by Fig. 3 in which the probability distributions of the single-cell $\lambda$ values are illustrated for each one of the tested strains. As demonstrated by the comparative evaluation of the distributions corresponding to the Control and MS treatments, the way in which the presence of microbial supernatant affected the position and the shape of the single-cell $\lambda$ distributions was straindependent (Fig. 3). Given the widely recognized importance of singlecell dynamics for quantitative microbial risk assessment (Ross and McMeekin, 2003), great attention has been drawn recently to the need of evaluating the behavior of single cells (or small populations/ sub-populations) and describing its variability via the use of stochastic modeling approaches (Alonso et al., 2014; Koutsoumanis and Lianou, 2013). To our knowledge, this is the first study assessing the potential effect of QS signal molecules on the growth behavior of foodborne pathogens at the single-cell level.

A widely used methodology was applied in this study for the purpose of single-cell $\lambda$ estimation, according to which the number of cells per microplate well is estimated using the Poisson distribution, based on the number of negative samples (i.e. wells not showing growth) (Aguirre et al., 2011; Baranyi and Pin, 1999; Baranyi et al., 2009). Despite its undoubtedly significant advantages, including its simplicity and high effectiveness in the study of microbial kinetics, there are certain limitations of the applied methodology that need to be taken into account. Such limitations include the potential error introduced in $\lambda$ estimation by the wells showing growth originating from more than one cell, the assumption made that bacterial cells are only present in wells in which growth is detected, as well as the fact that the applied microbial supernatant may affect not only cell division but also cell size. Indicative of the potential uncertainty of single-cell $\lambda$ estimation in this study is Fig. 4. When the CV of $\lambda$ was calculated for each experimental replicate separately, it was observed that in some cases (i.e. MS treatment in S. Typhimurium and St. aureus), the single-cell variability of this parameter was considerably different among replicates, as indicated by the high standard deviation values (Fig. 4). Nonetheless, replicating the experimental trials is inevitable in the context of the applied

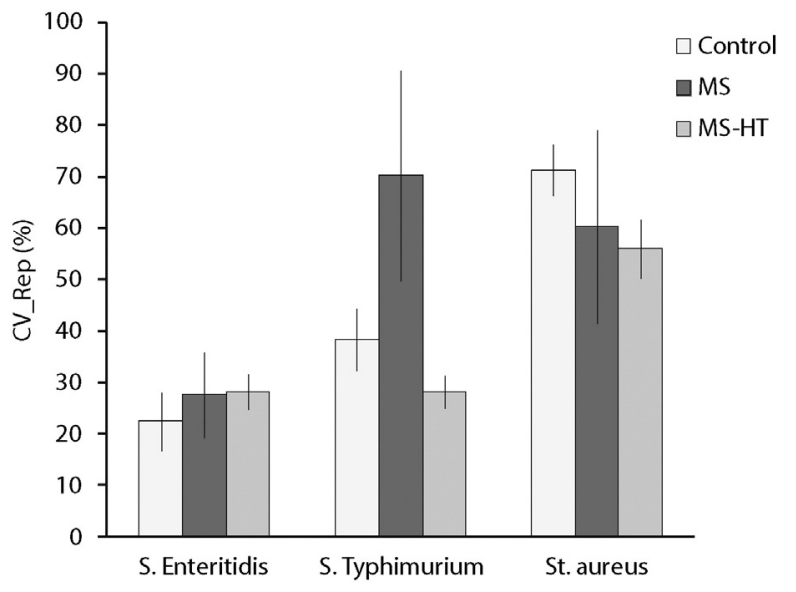

Fig. 4. Mean ( \pm standard deviation) coefficient of variation of the single-cell lag time values of the tested pathogens' strains (i.e. Salmonella enterica ser. Enteritidis, S. enterica ser. Typhimurium and Staphylococcus aureus), in the absence (Control) or presence of $20 \%(v / v)$ microbial supernatant (MS) or microbial supernatant subjected to heat treatment (MS-HT), estimated separately for each experimental replicate (CV_Rep).

methodology since large data sets are needed in order for the single-cell variability of $\lambda$ to be properly assessed.

At this point it should be noted that the contribution to the observations made of other (than or in addition to AI-2) signal molecules, or even of unknown non-signaling compounds (e.g., metabolic products), that are also present in the microbial supernatant, cannot be excluded. Despite the fact that the MS-HT treatment was applied as a supplementary control treatment, the conflicting results obtained with regard to the estimated $\lambda$ values limited its value. In contrast to what would be expected, the single-cell $\lambda$ of $S$. Typhimurium and St. aureus was significantly $(P \leq 0.05)$ longer in the MS-HT than in the Control (Table 3, Fig. 3). Although, in agreement with previous findings (Chorianopoulos et al., 2010), GC-MS and HPLC analyses of the tested media (Control, MS and MS-HT) did not reveal any considerable differences in their composition with regard to volatile compounds and organic acids (data not shown), differences may exist in other chemical compounds. For instance, inactivation of enzymes interfering in the tested system or production of toxic by-products (e.g., non-enzymatic browning reaction compounds) in response to the applied heat treatment may be potential explanations for the obtained results. The inconclusiveness of the observations made with regard to the MS-HT treatment, necessitates the design and conductance of additional experiments, if the exact role of QS on the metabolic responses and growth phenotype of the tested organisms is to be ascertained. Such experiments should be part of a more refined and basic research which, based on the results of the present study, will also involve the use of a
(A)

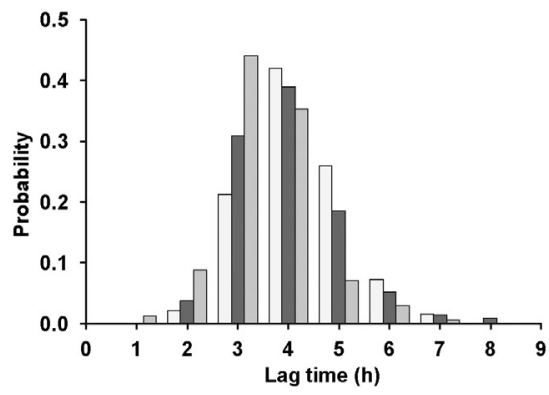

(B)

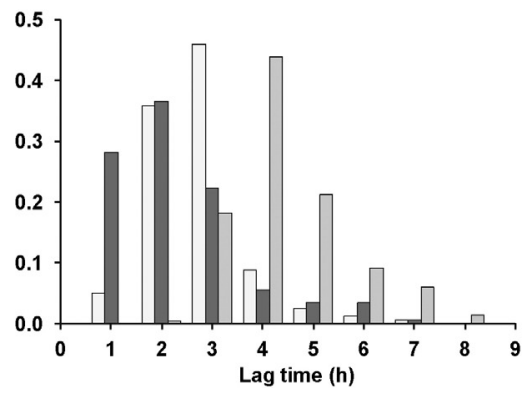

(C)

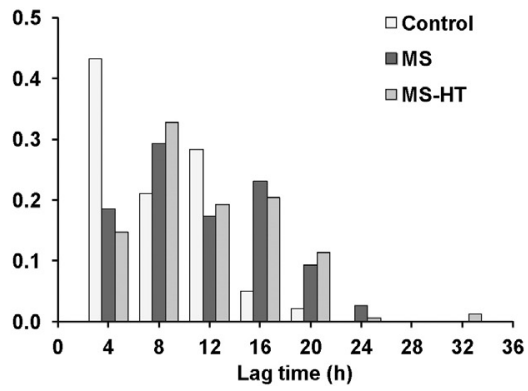

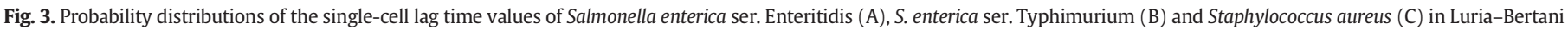

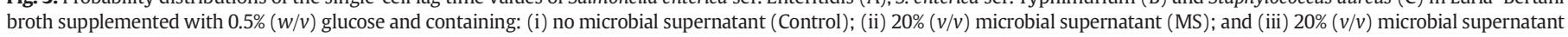
that was subjected to heat treatment (MS-HT). 
luxS-mutant of the tested $S$. Typhimurium strain (Jesudhasan et al., 2010) and/or inhibitors of AI-2 signaling (Guo et al., 2013).

In conclusion, as demonstrated by the results of this study, the effect of a $S$. Typhimurium culture supernatant, exhibiting AI-2 activity, on the single-cell lag time of S. enterica and St. aureus and its variability depended on the tested bacterial strain. Although preliminary in nature, the collected data should provide a good basis for future research on the role of QS in the single-cell growth behavior of foodborne pathogens, knowledge that is, in turn, expected to be useful in the control of such pathogens in situ.

\section{Acknowledgments}

We acknowledge the action THALIS: "Biological Investigation Of the Forces that Influence the Life of pathogens having as Mission to Survive in various Lifestyles; BIOFILMS". The action has been co-financed by the European Union (European Social Fund - ESF) and Greek national funds through the Operational Program "Education and Lifelong Learning" of the National Strategic Reference Framework (NSRF) - Research Funding Program: THALES. Investing in knowledge society through the ESF.

We are grateful to Dr. Sophia Kathariou (North Carolina State University, Raleigh, NC) for kindly providing the methicillin-resistant St. aureus strain, and to Aggeliki Kotzia and Fotini Pavli for their valuable technical assistance.

\section{References}

Aguirre, J.S., Rodríguez, M.R., García de Fernando, G.D., 2011. Effects of electron beam irradiation on the variability in survivor number and duration of lag phase of four food-borne organisms. Int. J. Food Microbiol. 149, 236-246.

Alonso, A.A., Molina, I., Theodoropoulos, C., 2014. Modeling bacterial population growth from stochastic single-cell dynamics. Appl. Environ. Microbiol. 80, 5241-5244.

Atkinson, S., Williams, P., 2009. Quorum sensing and social networking in the microbial world. J. R. Soc. Interface 6, 959-978.

Baranyi, J., Pin, C., 1999. Estimating bacterial growth parameters by means of detection times. Appl. Environ. Microbiol. 65, 732-736.

Baranyi, J., George, S.M., Kutalik, Z., 2009. Parameter estimation for the distribution of single cell lag times. J. Theor. Biol. 259, 24-30.

Bassler, B.L., Greenberg, E.P., Stevens, A.M., 1997. Cross-species induction of luminescence in the quorum-sensing bacterium Vibrio harveyi. J. Bacteriol. 179, 4043-4045.

Bertani, G., 1951. Studies on lysogenesis. I. The mode of phage liberation by lysogenic Escherichia coli. J. Bacteriol. 62, 293-300.

Blana, V.A., Nychas, G.-J.E., 2014. Presence of quorum sensing compounds in minced beef stored under various temperature and packaging conditions. Int. J. Food Microbiol. $173,1-8$.

Blana, V.A., Doulgeraki, A.I., Nychas, G.-J.E., 2011. Autoinducer-2-like activity in lactic acid bacteria isolated from minced beef packaged under modified atmospheres. J. Food Prot. 74, 631-635.

Chorianopoulos, N.G., Giaouris, E.D., Kourkoutas, Y., Nychas, G.-J.E., 2010. Inhibition of the early stage of Salmonella enterica serovar enteritidis biofilm development on stainless steel by cell-free supernatant of a Hafnia alvei culture. Appl. Environ. Microbiol. 76, 2018-2022.

Dourou, D., Ammor, M.S., Skandamis, P.N., Nychas, G.-J.E., 2011. Growth of Salmonella enteritidis and Salmonella typhimurium in the presence of quorum sensing signaling compounds produced by spoilage and pathogenic bacteria. Food Microbiol. 28, 1011-1018.

Duan, K., Silbey, C.D., Davidson, C.J., Surette, M.G., 2009. Chemical interactions between organisms in microbial communities. Contrib. Microbiol. 16, 1-17.

Dunstall, G., Rowe, M.T., Wisdom, G.B., Kilpatrick, D., 2005. Effect of quorum sensing agents on the growth kinetics of Pseudomonas spp. of raw milk origin. J. Dairy Res. 72, 276-280.

Federle, M.J., Bassler, B.L., 2003. Interspecies communication in bacteria. J. Clin. Investig. $112,1291-1299$.
Fuqua, W.C., Winans, S.C., Greenberg, E.P., 1994. Quorum sensing in bacteria: the LuxRlux-I family of cell density-responsive transcriptional regulators. J. Bacteriol. 176 269-275.

Gill, S.R., Fouts, D.E., Archer, G.L., Mongodin, E.F., Deboy, R.T., Ravel, J., Paulsen, I.T., Kolonay, J.F., Brinkac, L., Beanan, M., Dodson, R.J., Daugherty, S.C., Madupu, R. Angiuoli, S.V., Durkin, A.S., Haft, D.H., Vamathevan, J., Khouri, H., Utterback, T., Lee C., Dimitrov, G., Jiang, L., Qin, H., Weidman, J., Tran, K., Kang, K., Hance, I.R., Nelson, K.E., Fraser, C.M., 2005. Insights on evolution of virulence and resistance from the complete genome analysis of an early methicillin-resistant Staphylococcus aureus strain and a biofilm-producing methicillin-resistant Staphylococcus epidermidis strain. J. Bacteriol. 187, 2426-2438.

Guo, M., Gamby, S., Zheng, Y., Sintim, H.O., 2013. Small molecule inhibitors of AI-2 signaling in bacteria: state-of-the-art and future perspectives for anti-quorum sensing agents. Int. J. Mol. Sci. 14, 17694-17728.

Hoiseth, S.K., Stocker, B.A., 1981. Aromatic-dependent Salmonella typhimurium are nonvirulent and effective as live vaccines. Nature 291, 238-239.

Jesudhasan, P.R., Cepeda, M.L., Widmer, K., Dowd, S.E., Soni, K.A., Hume, M.E., Zhu, J., Pillai, S.D., 2010. Trancriptome analysis of genes controlled by luxS/autoinducer-2 in Salmonella enterica serovar typhimurium. Foodborne Pathog. Dis. 7, 399-410.

Kolling, G.L., Matthews, K.R., 2007. Influence of enteric bacteria conditioned media on recovery of Escherichia coli 0157:H7 exposed to starvation and sodium hypochlorite. J. Appl. Microbiol. 103, 1435-1441.

Kong, K.-F., Vuong, C., Otto, M., 2006. Staphylococcus quorum sensing in biofilm formation and infection. Int. J. Med. Microbiol. 296, 133-139.

Koutsoumanis, K.P., Lianou, A., 2013. Stochasticity in colonial growth dynamics of individual bacterial cells. Appl. Environ. Microbiol. 79, 2294-2301.

Lianou, A., Koutsoumanis, K.P., 2011. A stochastic approach for integrating strain variability in modeling Salmonella enterica growth as a function of $\mathrm{pH}$ and water activity. Int J. Food Microbiol. 149, 254-261.

Lopes, S.P., Machado, I., Pereira, M.O., 2011. Role of planktonic and sessile extracellular metabolic byproducts on Pseudomonas aeruginosa and Escherichia coli intra and interspecies relationships. J. Ind. Microbiol. Biotechnol. 38, 133-140.

Lu, L., Hume, M.E., Pillai, S.D., 2004. Autoinducer-2-like activity associated with foods and its interaction with food additives. J. Food Prot. 67, 1457-1462.

Nadell, C.D., Xavier, J.B., Levin, S.A., Foster, K.R., 2008. The evolution of quorum sensing in bacterial biofilms. PLoS Biol. 6, 171-179.

Nychas, G.-J.E., Dourou, D., Skandamis, P., Koutsoumanis, K., Baranyi, J., Sofos, J., 2009. Effect of microbial cell-free meat extract on the growth of spoilage bacteria. J. Appl. Microbiol. 107, 1819-1829.

Painter, K.L., Krishna, A., Wingeshweraraj, S., Edwards, A.M., 2014. What role does the quorum-sensing accessory gene regulator system play during Staphylococcus aureus bacteremia? Trends Microbiol. 22, 676-685.

Oin, Z., Yang, L., Qu, D., Molin, S., Tolker-Nielsen, T., 2009. Pseudomonas aeruginosa extracellular products inhibit staphylococcal growth, and disrupt established biofilms produced by Staphylococcus epidermidis. Microbiology 155, 2148-2156.

Rasch, M., Andersen, J.B., Nielsen, K.F., Flodgaard, L.R., Christensen, H., Giskov, M., Gram, L. 2005. Involvement of bacterial quorum-sensing signals in spoilage of bean sprouts. Appl. Environ. Microbiol. 71, 3321-3330.

Ross, T., McMeekin, T.A., 2003. Modeling microbial growth within food safety risk assessments. Risk Anal. 23, 179-197.

Skandamis, P.N., Nychas, G.-J.E., 2012. Quorum sensing in the context of food microbiology. Appl. Environ. Microbiol. 78, 5473-5482.

Surette, M.G., Bassler, B.L., 1998. Quorum sensing in Escherichia coli and Salmonella typhimurium. Proc. Natl. Acad. Sci. U. S. A. 95, 7046-7050.

Taga, M.E., Miller, S.T., Bassler, B.L., 2003. Lsr-mediated transport and processing of AI-2 in Salmonella typhimurium. Mol. Microbiol. 50, 1411-1427.

Tindall, B., Grimont, P., Garrity, G., Euzeby, J., 2005. Nomenclature and taxonomy of the genus Salmonella. Int. J. Syst. Evol. Microbiol. 55, 521-524.

Waters, C.M., Bassler, B.L., 2005. Quorum sensing: cell-to-cell communication in bacteria. Annu. Rev. Cell Dev. Biol. 21, 319-346.

Wevers, E., Moons, P., Van Houdt, R., Lurquin, I., Aertsen, A., Michiels, C.W., 2009. Quorum sensing and butanediol fermentation affect colonization and spoilage of carrot slices by Serratia plymuthica. Int. J. Food Microbiol. 134, 63-69.

Whan, L., Dunstall, G., Rowe, M.T., 2000. A study of the growth kinetics of two pseudomonads from pasteurized milk and the possible role of quorum sensing. Milchwissenschaft 55, 371-373.

Yu, D., Zhao, L., Xue, T., Sun, B., 2012. Staphylococcus aureus autoinducer-2 quorum sensing decreased biofilm formation in an icaR-dependent manner. BMC Microbiol. 12, 288-300. 